\title{
Prevalencia de lesión nerviosa periférica por fractura supracondílea de húmero en pacientes pediátricos
}

\author{
Prevalence of peripheral nervous injury due to supracondyleal \\ fracture of the humerus in pediatric patients \\ Jesús Ruiz-Ruvalcaba, ${ }^{*}$ Pablo Espinoza-Hernández, ${ }^{\ddagger}$ Carlos Cuevas-Mora§ \\ *Médico Residente del cuarto año de Traumatología y Ortopedia; ${ }^{\ddagger}$ Médico adscrito al Servicio de Traumatología y Ortopedia; $\$$ Médico \\ Traumatólogo y Ortopedista. Hospital General de Zona No. 16, Torreón, Coahuila.
}

\begin{abstract}
Resumen
Introducción: Las fracturas supracondíleas de húmero son las más frecuentes del codo en niños (50-60\%), con pico máximo de 5-8 años, distribución 2:1 en hombres y predilección por el lado no dominante. Se producen por mecanismos directos e indirectos (95\%). Pueden presentar lesión neurológica asociada en 5-19\%. Objetivo: Identificar la prevalencia de lesión nerviosa por fractura supracondílea de húmero en pacientes pediátricos. Material y métodos: Captamos pacientes pediátricos con fractura supracondílea Gartland III atendidos en el periodo de marzo de 2020 a enero de 2021, fueron explorados en busca de lesión del nervio radial, mediano y cubital al momento de la atención primaria en urgencias y a los dos meses. Resultados: Se incluyeron 26 pacientes como muestra final. Se observó que 23\% presentaron datos clínicos de lesión nerviosa en fracturas supracondíleas humerales. Conclusión: Se demostró que la edad de presentación, sexo, incidencia y recuperación de la lesión nerviosa en miembro torácico producida en fracturas supracondíleas de húmero en niños en nuestro hospital son similares a los diferentes parámetros evaluados en centros de alta concentración.
\end{abstract}

Palabras clave: Supracondílea, lesión nerviosa, neuropraxia.

\begin{abstract}
Introduction: Supracondylar humerus fractures represent the most frequent of the elbow in children (50-60\%), with a maximum peak of 5-8 years, 2:1 distribution in males and predilection for the non-dominant side. They are produced by direct and indirect mechanisms (95\%). It can present associated neurological injury in 5-19\%. Objective: To identify the prevalence of nerve injury due to supracondylar humerus fracture in pediatric patients. Material and methods: We captured pediatric patients with a Gartland III supracondylar fracture treated in the period from March 2020 to January 2021, they were explored in search of injury to the radial, median and ulnar nerves at the time of primary care in the emergency room and at 2 months. Results: 26 patients were included as a final sample. Observing that $23 \%$ presented clinical data of nerve injury in supracondylar humeral fractures. Conclusion: It was demonstrated that the age of presentation, gender, incidence and recovery of nerve injury in the thoracic limb produced in supracondylar humerus fractures in children in our hospital are similar to the different parameters evaluated in high concentration centers.
\end{abstract}

Keywords: Supracondylar, nerve injury, neuropraxia.

\section{Introducción}

Las fracturas supracondíleas de húmero distal representan la segunda fractura más común en la infancia y las más frecuentes del codo en niños y adolescentes (50-60\%), tiene su pico máximo entre los 5-8 años, con una distribución entre hombre y mujer en relación 2:1 y presenta cierto grado de predilección por el lado no dominante. ${ }^{1}$

Esta lesión es definida como la pérdida de solución de continuidad en la metáfisis distal del
Correspondencia:

Jesús Ruiz-Ruvalcaba

E-mail: drjesusruizruv@ hotmail.com

Recibido: 28-04-2021. Aceptado: 05-05-2021.
Citar como: Ruiz-Ruvalcaba J, Espinoza-Hernández P, Cuevas-Mora C. Prevalencia de lesión nerviosa periférica por fractura supracondílea de húmero en pacientes pediátricos. Orthotips. 2021; 17 (3): 148-151. https://dx.doi.org/10.35366/100624 
húmero, por encima de los cóndilos y proximal a la línea fisaria, sitio que es más delgado por la localización de la fosa coronoidea y olecraneana. ${ }^{2}$

Este tipo de lesión puede producirse por dos mecanismos: directos (contusión con codo en flexión) e indirectos (carga axial con codo en extensión), siendo éste el más frecuente (95-97\%). ${ }^{3}$

Representan una emergencia médica debido a las posibles lesiones neurológicas (5-14\%) y vasculares asociadas (7-10\%). Una lesión neurológica puede ocurrir como una lesión primaria o secundaria, en la actualidad la más frecuente es la del nervio radial $(41.2 \%)$, seguido del nervio mediano (36\%) y por último el nervio cubital (22.8\%). ${ }^{4}$

Las lesiones primarias son el resultado del impacto energético inicial y el desplazamiento de la fractura, que causa estiramiento, atrapamiento o interrupción del nervio. En las fracturas de tipo de extensión el desplazamiento se describe con mayor frecuencia como posteromedial y causa lesiones del nervio radial; mientras que el desplazamiento posterolateral es más probable que ocasione una lesión del nervio mediano, siendo la neuropraxia la causa más frecuente de manifestación, y teniendo una recuperación espontánea dentro de los primeros cuatro meses. El nervio cubital es el más comúnmente lesionado en el tipo de flexión. Las lesiones secundarias pueden ocurrir como consecuencia de una manipulación excesiva. ${ }^{4,5}$

Se ha descrito compromiso neurológico, habitualmente neuropraxia, en 3 a $22 \%$ de los pacientes con fracturas supracondíleas. Se puede dañar cualquiera de los nervios periféricos (mediano, interóseo anterior, radial o cubital). ${ }^{5}$

La recuperación espontánea se demostró en una serie de casos de parálisis postoperatoria del nervio cubital. Se evidenció la remisión completa después de 49 días (rango 2-224 días) para el nervio interóseo anterior. También se observó recuperación espontánea del nervio mediano y radial. La mediana del tiempo hasta la recuperación nerviosa fue de 2.3 meses (rango 1.3-3.7 meses). Después de tres (60\%) y seis meses $(90 \%)$ los pacientes afectados experimentaron la recuperación de los nervios afectados. ${ }^{6}$

El Dr. José Piñeiro y cols. en su estudio de 360 fracturas supracondíleas tipo III manejadas con fijación cruzada encontraron las siguientes secuelas: lesión neurológica en 3.3\%, lesión vascular en $2.5 \%$, infecciones superficiales en $6.9 \%$, migración de clavos en $3.8 \%$, cúbito varo en $3.3 \%$, cúbito valgo en $2.0 \%$, defecto rotacional en $8.0 \%$, limitación flexo extensión en $1.0 \%{ }^{7}$
MacQuillan y cols. encontraron que un total de 166 nervios resultaron heridos en 196 niños; 26 tenían disfunción neurológica que afectaba a dos o más nervios. Por lo general, el nervio más lesionado fue el nervio cubital (43.4\%), seguido por el nervio mediano (36.7\%) y los nervios radiales (19.9\%). De los cuales 165 de ellos se presentaron por fractura en extensión y únicamente uno por fractura en flexión. ${ }^{8}$

Benjamin Shore y cols., en su estudio en el cual se valoró el tiempo de recuperación de la lesión nerviosa secundaria a fracturas supracondíleas, concluyeron que la mayoría de los niños con lesiones nerviosas motoras asociadas demostraron recuperación dentro de los seis meses posteriores a su lesión, y aquellos pacientes con lesiones del nervio radial o más de un nervio lesionado tienen un mayor riesgo de recuperación tardía. No recomiendan la exploración nerviosa aguda. 9,10

Es habitual la recuperación completa de la función nerviosa, aunque esto puede requerir varios meses. Algunos autores recomiendan la exploración quirúrgica si la función nerviosa no se ha recuperado a las 6-8 semanas de la reducción, mientras que según otros autores se deben dejar transcurrir un mínimo de 2-4 meses para la resolución. Las parálisis mantenidas después de la consolidación de la fractura pueden indicar el atrapamiento del nervio en el callo. ${ }^{11,12}$

El objetivo es identificar la prevalencia de lesión nerviosa por fractura supracondílea de húmero en pacientes pediátricos, en el periodo de marzo de 2020 a enero de 2021.

\section{Material y métodos}

El presente estudio se realizó en el Servicio de Ortopedia Pediátrica y se revisaron 26 pacientes con diagnóstico de fractura supracondílea humeral, en el periodo comprendido de marzo de 2020 a enero de 2021; se captó un total de 32 pacientes, de los cuales con base en una serie de criterios de inclusión, exclusión y eliminación se dejó como muestra total la cantidad de 26 pacientes. Se realizó un estudio observacional, prospectivo, transversal, descriptivo.

El grupo de estudio está integrado por pacientes que cumplían con los criterios de inclusión, los cuales fueron: pediátricos de entre tres y 10 años, ambos sexos, con fractura supracondílea humeral tipo III de la clasificación de Gartland modificada por Wilkins, sin importar si se manejó con reducción cerrada o abierta, fijada con clavillos de Kirschner cruzados o laterales y que fueron tratados. Los criterios de exclusión fueron 
pacientes con fractura supracondílea humeral tipo I y II de la clasificación de Gartland modificada, con fracturas asociadas en la misma extremidad, edad fuera del rango, con lesión nerviosa en extremidad afectada previo al evento traumático. Mientras que los criterios de eliminación fueron pacientes que no deseaban participar en el estudio, que no contaban con expediente clínico completo y que no acudieron a su valoración a los 60 días.

Todos los pacientes se evaluaron en tres tiempos: el primero de ellos a su llegada al área de urgencias, previa reducción; el segundo, previo a su egreso hospitalario posterior a su intervención quirúrgica y el tercero fue en la consulta externa, dos meses después del evento inicial. La valoración fue puramente clínica y dirigida a valorar las diversas áreas tanto sensitiva como motora de los nervios periféricos de la siguiente manera:

Nervio radial: la función motora se valoró mediante la extensión del pulgar (extensor largo y el extensor corto). La función sensitiva se exploró en la superficie dorsal del dedo pulgar.

Nervio mediano: la función motora se evaluó con la oposición del pulgar (oponente del pulgar). La sensibilidad se exploró en la piel palmar de la punta del dedo índice.

Nervio cubital: la función motora se exploró con la aducción del pulgar (aductor del pulgar). La función sensitiva se evaluó en la superficie palmar de la punta del quinto dedo.

La recolección de datos se realizó a través de una tabla de captura de datos, las variables estudiadas fueron las siguientes: edad, sexo, lado lesionado y presencia de lesión nerviosa.

\section{Resultados}

Con base en el tamaño de muestra considerado, y mediante los criterios de inclusión, exclusión y eliminación del protocolo, se incluyeron finalmente a 26 pacientes, se realizó la captura de variables del expediente clínico, sobre las notas registradas en el seguimiento de pacientes, según los datos obtenidos del presente estudio y bajo los objetivos propuestos utilizados para el análisis de variables, se encontraron los siguientes resultados:

Con relación al sexo encontramos un predominio de pacientes masculinos con 17 casos (65\%) contra nueve $(35 \%)$ del sexo femenino.

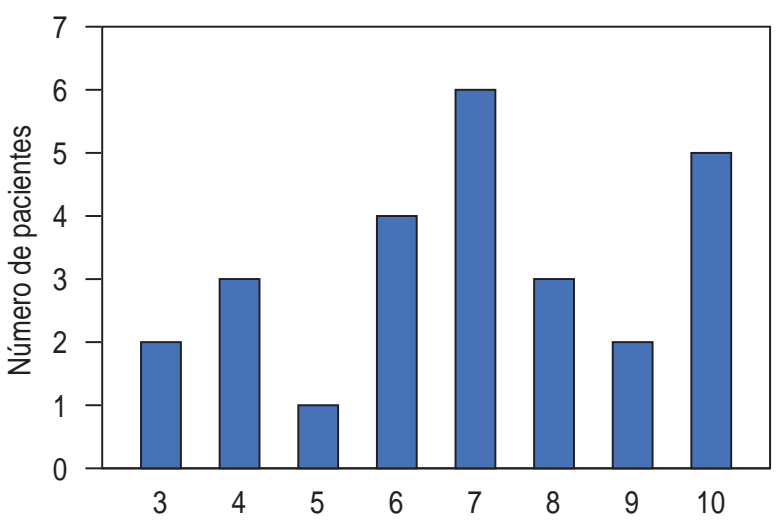

Figura 1: Edad de presentación.

Con respecto a la edad encontramos pacientes entre los tres y 10 años, con un promedio de 6.9, ubicándose la mayor frecuencia a los siete años con seis casos (Figura 1).

De acuerdo con el miembro lesionado, se encontró que el dominante es el más frecuentemente afectado con 15 casos (58\%), 11 tuvieron afección del miembro no dominante.

Mediante la exploración clínica inicial se buscó intencionadamente la presencia de lesión nerviosa (sensitiva y/o motora) del nervio radial, mediano y cubital, se encontró que $76.92 \%$ no presenta lesión nerviosa, mientras que $23.08 \%$ sí la presenta, la más frecuente fue la del nervio radial, representando $66.64 \%$, seguido de $16.68 \%$ para el nervio mediano y cubital, respectivamente.

Dentro de los seis pacientes que presentaron lesión nerviosa la distribución fue: tres con lesión sensitiva nervio radial $50 \%$ y motora $16 \%$, lesión sensitiva del nervio mediano $17 \%$ y del nervio cubital sensitivo 17\%.

Del mismo modo, a los dos meses posteriores a la reducción y fijación se exploró a los pacientes que presentaron lesión nerviosa mediante las mismas maniobras de exploración y se encontró que 100\% presentó recuperación total de la función normal.

\section{Discusión}

Los reportes en la literatura muestran evidencia de lesión neurológica en aproximadamente 5 a 14\%. En la actualidad, la más frecuente es la del nervio radial $(41.2 \%)$, seguido del nervio mediano (36\%) y por último el nervio cubital (22.8\%). Asimismo, autores como D'Ambrosia, Lipscon y Randall en sus estudios reportan porcentajes similares. 
En el presente estudio se demostró que la prevalencia de lesión nerviosa en las fracturas supracondíleas humerales en los niños estudiados fue de $23.1 \%$. De los cuales el nervio radial fue el más afectado, ocupando $66.64 \%$, seguido por el nervio mediano y cubital con $16.68 \%$ cada uno.

A su vez se encontró que la principal afección nerviosa fue sensitiva, ya que de los seis pacientes que presentaban lesión nerviosa, cuatro tenían lesión del nervio radial (tres sensitivas y una motora), uno del mediano y uno del cubital, dando un total de seis lesiones encontradas. Mientras que sólo se vio afección motora en un paciente y ésta fue en el nervio radial.

Se corrobora que la lesión neurológica más usual es la del nervio radial, con base en que el mecanismo de acción de la fractura (carga axial con miembro en extensión) es el más frecuente en presentarse (95\%), a su vez el desplazamiento posteromedial del fragmento es el causante de la mayor afectación del nervio radial, con respecto al mediano, esto causado por la posición de la extremidad al momento de la carga axial.

De los pacientes en los que se reportó lesión nerviosa inicial, $100 \%$ logró una recuperación total en un periodo de dos meses, independientemente de si la lesión inicial fue sensitiva, motora o mixta. No se contó con el acceso a estudios avanzados para diferenciar el tipo de lesión nerviosa, pero con base en los antecedentes y en la evolución de la misma inferimos que se trató de una neuropraxia.

\section{Conclusiones}

Dentro del objetivo del presente estudio se demostró la prevalencia de la lesión nerviosa en miembro torácico producida en fracturas supracondíleas de húmero en infantes. Se encontraron datos muy similares a los descritos en la mayoría de la literatura revisada en la elaboración de este estudio, con una varianza mínima de las cifras, así se pudo corroborar que las cifras descritas en los diferentes parámetros evaluados en centros de alta concentración son similares a las de la población pediátrica en nuestro hospital.

\section{Referencias}

1. Omid R, Choi PD, Skaggs DL. Supracondylar humeral fractures in children. J Bone Joint Surg Am. 2008; 90 (5): 1121-1132. doi: 10.2106/JBJS.G.01354.
2. Pimienta PN, Castillo HE, Matos OJL, Aguila CLR, Ballate $\mathrm{MD}$, Alfonso CY. Incidencia de las fracturas supracondíleas del codo en Pediatría: fijación percutánea lateral. Acta Med Cent. 2018; 12 (2): 140-147. doi: 10.11622/smedj.2017094.

3. Harris LR, Arkader A, Broom A, Flynn J, Yellin J, Whitlock P, et al. Pulseless supracondylar humerus fracture with anterior interosseous nerve or median nerve injury-an absolute indication for open reduction? J Pediatr Orthop. 2019; 39 (1): e1-e7. doi: 10.1097/BPO.0000000000001238.

4. Flynn K, Shah AS, Brusalis CM, Leddy K, Flynn JM. Flexiontype supracondylar humeral fractures: ulnar nerve injury increases risk of open reduction. J Bone Joint Surg Am. 2017; 99 (17): 1485-1487. doi: 10.2106/JBJS.17.00068.

5. Valencia M, Moraleda L, Díez-Sebastián J. Long-term functional results of neurological complications of pediatric humeral supracondylar fractures. J Pediatr Orthop. 2015; 35 (6): 606-610. doi: 10.1097/BPO.0000000000000337.

6. Rupp M, Schafer C, Heiss C, Alt V. Pinning of supracondylar fractures in children - Strategies to avoid complications. Injury. 2019; 50 Suppl 1: S2-S9. doi: 10.1016/j. injury.2019.03.042.

7. Piñeiro J. Tratamiento de las fracturas supracondíleas en el Hospital de Niños de Corrientes. Rev Asoc Arg Ortop y Traumatol. 1996; 61 (3): 322-328.

8. Kwok IH, Silk ZM, Quick TJ, Sinisi M, MacQuillan A, Fox M. Nerve injuries associated with supracondylar fractures of the humerus in children: our experience in a specialist peripheral nerve injury unit. Bone Joint J. 2016; 98-B (6): 851-856.

9. Shore BJ, Gillespie BT, Miller PE, Bae DS, Waters PM. Recovery of motor nerve injuries associated with displaced, extension-type pediatric supracondylar humerus fractures. J Pediatr Orthop. 2019; 39 (9): e652-e656. doi: 10.1097/ BPO.0000000000001056.

10. Granier C, Maury E, Coulet B, Delpont M, Cottalorda J, Sleth $C$, et al. Median nerve entrapment after supracondylar humeral fracture in a young child. Case Rep Orthop. 2019; 2019: 1543126. doi: 10.1155/2019/1543126.

11. Dolan RT, Giele HP. Radial nerve palsies associated with paediatric supracondylar humeral fractures: a caution in the interpretation of neurophysiological studies. J Pediatr Orthop B. 2020; 29 (2): 126-132. doi: 10.1097/ BPB. 0000000000000680 .

12. Bertelli J, Soldado F, Ghizoni MF. Outcomes of radial nerve grafting in children after distal humerus fracture. J Hand Surg Am. 2018; 43 (12): 1140.e1-1140.e6. doi: 10.1016/j. jhsa.2018.04.018.

\section{Conflicto de intereses}

Los autores declaran no tener conflicto de intereses con respecto a este trabajo.

No se han realizado experimentos en seres humanos ni animales.

Los autores declaran que en este artículo no aparecen datos de pacientes. 